\title{
Oral antikoagulieren oder die Plättchen hemmen?
}

Fragestellung: Gibt es einen Unterschied zwischen einer Behandlung mit Vitamin-K-Antagonisten wie Warfarin und Acetylsalicylsäure bei Patienten mit Herzinsuffizienz im Sinusrhythmus?

Hintergrund: Die chronische Herzinsuffizienz ist ein zunehmendes Gesundheitsproblem, auch in Deutschland. Patienten mit Herzinsuffizienz haben ein erhöhtes Risiko zerebraler Embolien. Patienten mit Herzinsuffizienz haben auch ein erhöhtes Risiko des plötzlichen Herztodes. Dies erklärt, warum viele Leitlinien, obwohl es keine wissenschaftliche Evidenz gibt, den Einsatz von Acetylsalicylsäure oder oralen Antikoagulanzien in dieser Patientengruppe empfehlen.

Patienten und Methodik: Die WARCEF-Studie war eine randomisierte Studie bei Patienten mit Herzinsuffizienz im Sinusrhythmus. Die eine Hälfte der Studienteilnehmer wurde auf eine orale Antikoagulation mit dem Vitamin-K-Antagonisten Warfarin eingestellt, mit einem INR-Zielbereich zwischen 2 und 3,5. Die andere Hälfte der Patienten wurde mit 325 mg Acetylsalicylsäure pro Tag behandelt. Die Studie war doppelblind, multizentrisch und randomisiert. Eingeschlossen wurden Patienten im Alter über 18 Jahren mit einer linksventrikulären Ejektionsfraktion von $35 \%$ oder weniger. Patienten mit Vorhofflimmern wurden ausgeschlossen.

Der primäre Endpunkt war die Zeit bis zum Auftreten eines ersten ischämischen Insults, einer intrazerebralen Blutung oder Tod.

Ergebnisse: In die Vitamin-K-Antagonisten-Gruppe wurden 1.142 Patienten und in die Acetylsalicylsäure-Gruppe 1.163 Patienten eingeschlossen. Das mittlere Alter betrug 61 Jahre und $80 \%$ der Patienten waren männlich. Die mittlere Ejektionsfraktion lag bei $25 \%$.

Der primäre Endpunkt wurde von 302 Patienten der VitaminK-Antagonisten-Gruppe und 320 Patienten der Acetylsalicylsäure-Gruppe erreicht; dies entspricht einer Ereignisrate von 7,47 pro 100 Patientenjahre für die orale Antikoagulation mit Warfarin und 7,93 für die plättchenhemmende Therapie mit Acetylsalicylsäure ( Abb. 1). Die Hazard Ratio (HR) betrug 0,93 und war statistisch nicht signifikant.

Es ergaben sich keine Unterschiede für Tod und intrazerebrale Blutungen. Ischämische Insulte waren allerdings mit 29 versus

Homma S, Thompson JL, Pullicino PM et al, WARCEF Investigators. Warfarin and aspirin in patients with heart failure and sinus rhythm. N Engl J Med 2012; 366: 1859-69 55 unter dem Vitamin-K-Antagonisten signifikant seltener. Auch für den kombinierten Endpunkt - Tod, ischämischer Insult, intrazerebrale Blutung oder intrakranielle Blutung ergab sich mit 307 mit dem Vitamin-K-Antagonisten versus

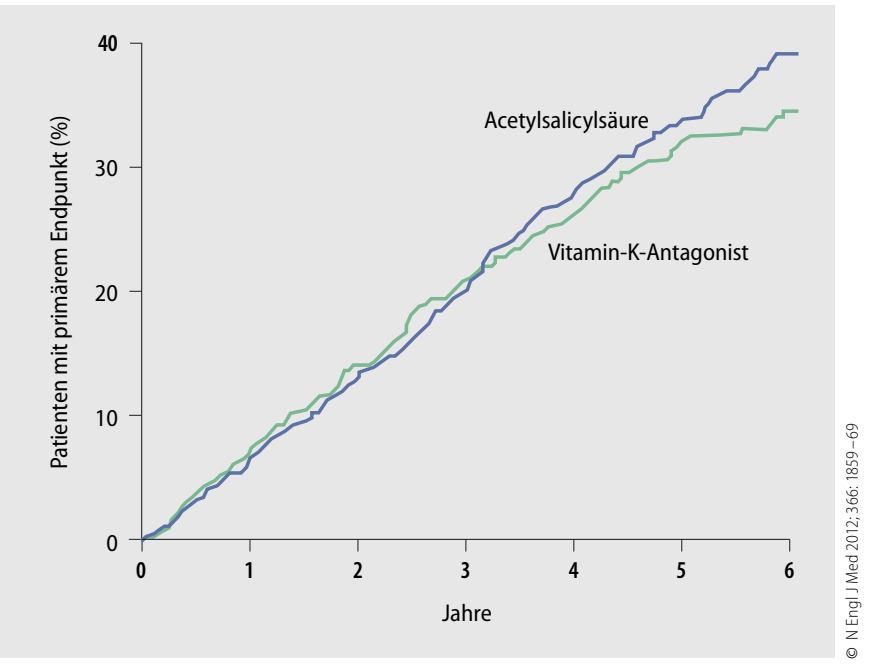

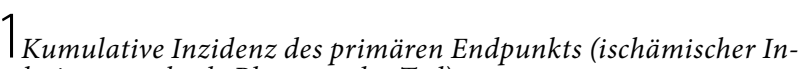
sult, intrazerebrale Blutung oder Tod).

323 mit Acetylsalicylsäure kein statistisch signifikanter Unterschied.

Schlussfolgerungen: Bei Patienten mit Herzinsuffizienz, die einen Sinusrhythmus aufweisen, gibt es weder im Hinblick auf primäre Ereignisse, noch auf Blutungskomplikationen Unterschiede zwischen einer oralen Antikoagulation mit dem Vitamin-K-Antagonisten Warfarin und einer Thrombozyten-funktionshemmenden Therapie mit Acetylsalicylsäure. Das reduzierte Risiko eines ischämischen Insults unter der oralen Antikoagulation wird durch die höhere Zahl von intrazerebralen Blutungen aufgehoben.

\section{- Kommentar von Prof. Hans-Christoph Diener \\ Antikoagulation nicht notwendig}

Die WARCEF-Studie beantwortet eine sehr wichtige wissenschaftliche Frage, nämlich, dass es keine Notwendigkeit gibt, Patienten mit Herzinsuffizienz und Sinusrhythmus zu antikoagulieren. Es ergeben sich keine klinisch relevanten Unterschiede zwischen den beiden Behandlungsgruppen. Allerdings beantwortet die WARCEF-Studie eine weitere sehr wichtige wissenschaftliche Frage nicht, nämlich, ob es überhaupt notwendig ist, diese Patienten mit Acetylsalicylsäure zu behandeln. Angesichts der nicht unerheblichen Zahl der Blutungskomplikationen müsste dies in einer weiteren Placebokontrollierten Studie im Vergleich zu Acetylsalicylsäure untersucht werden. 